University of Nebraska - Lincoln

DigitalCommons@University of Nebraska - Lincoln

Copyright, Fair Use, Scholarly Communication, etc.

2020

Accept me, accept me not: What do journal acceptance rates really mean?

Rachel Herbert

Follow this and additional works at: https://digitalcommons.unl.edu/scholcom

Part of the Intellectual Property Law Commons, Scholarly Communication Commons, and the Scholarly Publishing Commons

This Article is brought to you for free and open access by the Libraries at University of Nebraska-Lincoln at DigitalCommons@University of Nebraska - Lincoln. It has been accepted for inclusion in Copyright, Fair Use, Scholarly Communication, etc. by an authorized administrator of DigitalCommons@University of Nebraska Lincoln. 


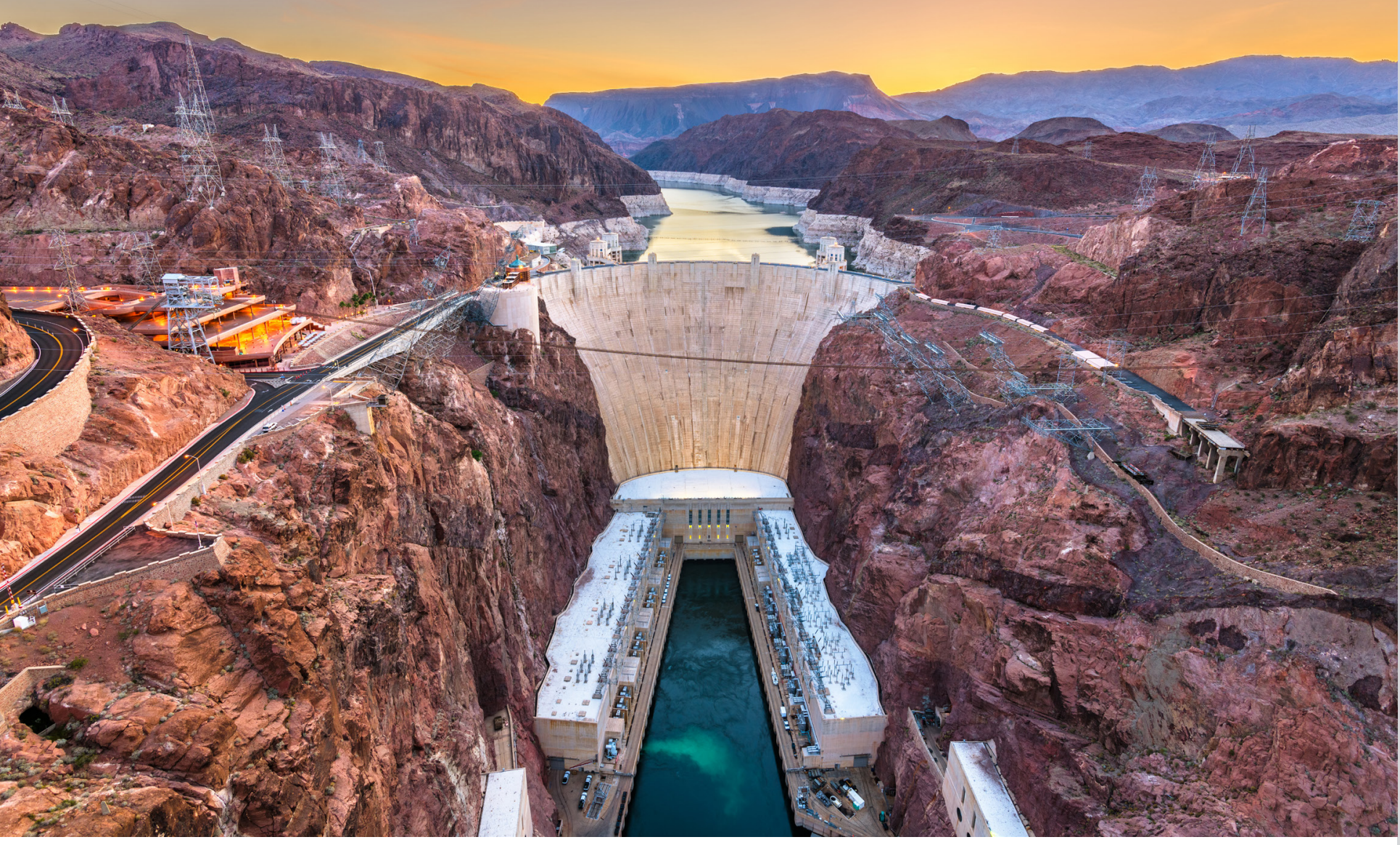

\section{Accept me, accept me not: What do journal acceptance rates really mean?}

\section{Highlights}

Journal acceptance rates should not be used as evaluative metrics for journals: we find no evidence that acceptance rates are a reliable signal of quality or impact.

Journal acceptance rates are useful for submitting authors and ICSR recommends that they be made publicly available where possible.

Gold open access journals do tend to have lower acceptance rates than other open access types, but these also tend to be younger journals: as these journals age, will those acceptance rates increase, or will the open access model influence the acceptance rate? 


\section{Introduction}

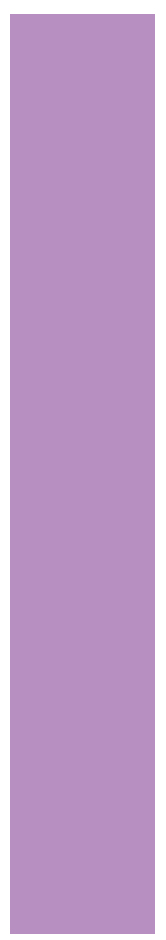

One of the functions performed by a journal is often called 'gate-keeping': the selection of research which is deemed worthy of publication. Selecting from unsolicited manuscripts submitted to the journal, the editorial team may choose to accept some and reject others (often after peer review). The acceptance rate-the proportion of manuscripts that are selected for publication from the pool of all submitted manuscripts-is an apparently straightforward measure that appears quite regularly on journal metric pages (e.g. Elsevier Journal Insights), in editor reports (e.g. Hall, 2018; Barber, 2019; Manning, 2017) and via journal finder tools (e.g. Elsevier Journal Finder, Springer Journal Suggester). Does an acceptance rate have any meaning as an evaluative metric, though?
The definition:

Journal Acceptance Rate $=$ Accepted Manuscripts / Submitted Manuscripts

Wait, there's another option! Publishers and editors reading this may also be familiar with another form of acceptance rate: Accepted Manuscripts / (Accepted + Rejected Manuscripts). Both indicators measure a rate of acceptances, but with a different approach. In this second definition (we'll call it Type II), it is the share of decisions that is calculated. Type II will be useful particularly for those monitoring the peer review process closelymost typically publishers and editors. The former definition (we'll call that Type I) is the definition most commonly reported on journal and publisher webpages. We believe this is the definition of Journal Acceptance Rates that authors interact with and will benefit from, so that's why it is Type I that we use throughout this report.

\section{What do acceptance rates measure?}

The number of accepted manuscripts might be expected to be driven by several factors: the quality, interest or importance of submitted manuscripts, the number of and relationships to other journals in the same field, and perhaps any submission or peer review backlogs or page limitations. There is anecdotal evidence that some journals will target a particular acceptance rate. Each of these factors will have a varying impact on each journal's acceptance rate. The drivers of submission rates, on the other hand, are a little different: the size of the field-by volume of funding and number of researchers and publications, the number of and relationships among journals, journal 'brand' awareness or (perceived) prestige, and the potential impact of successful publication for the author (e.g. in some cases, being published in certain journals can help secure future research funding).

So if there are overlapping but different drivers for the two factors that determine an acceptance rate, what does the rate actually tell us about a journal? The Metrics Toolkit suggests that the rate can be used as a "proxy for perceived prestige and demand as compared to availability" (Metrics Toolkit). The impact of journals such as Nature and Science is talked about in relation toamong other things-their relatively low acceptance rates (e.g. Emmer, 2019). And at the other end of the spectrum, predatory journals are classified in a multitude of ways, but often that description includes mention of high acceptance rates (Forero, Oermann \& Manca, 2018; Brembs, 2018).

Yet the concept of separating the "wheat from the chaff" (Brembs, 2018) is pushed to the limit when journals like Nature and Science have acceptance rates of $10 \%$ or less. Being rejected from extremely selective journals surely can't tell us much about that manuscript. 
Those drivers we mentioned above signal many different 'problems' with a manuscript: one might be low quality or impact, another might be out of scope.

If articles cannot be judged on what journal they are rejected from because we cannot distinguish the reason for rejection, can a journal's acceptance rate really be a signal of quality or rigorousness of the peer review process? If not, then what does an acceptance rate really mean?

This research question has been tackled before, with journal acceptance or rejection rates compared to citation-based indicators and considered in relation to journal discipline, age and access type (e.g. Sugimoto, Larivière, Ni et al., 2013; Frontiers, 2015; Frontiers, 2016). However, the results conflict in some cases and journal rejection or acceptance rates remain linked to the idea of quality to some extent (Sugimoto, Larivière, Ni et al., 2013; Metrics Toolkit). It has also been suggested that rejection rates of "up to $30 \%$ are justifiable to ensure only sound research is published" (Frontiers, 2015).

And so we explored a set of 2,371 journals and their acceptance rates in 2017, the majority of which are published by Elsevier (see Methodology for full details). The journals represent a broad set of subject areas, journal types and ages; with all but the social sciences and arts and humanities wellrepresented, which is a limitation of the findings in this report. To understand what the acceptance rates of these journals might demonstrate, we have compared their acceptance rates to a variety of other indicators.
The dataset:

\section{2,371 journals}

of which $1,942(82 \%)$ are

published by Elsevier

2017 Journal Acceptance Rates

\section{An affection for rejection}

The journals in our dataset had acceptance rates ranging from $1.1 \%$ to $93.2 \%$ in 2017 , with an average of $32 \%$. We can see immediately that journals tend to accept fewer articles than they reject (Figure 1).

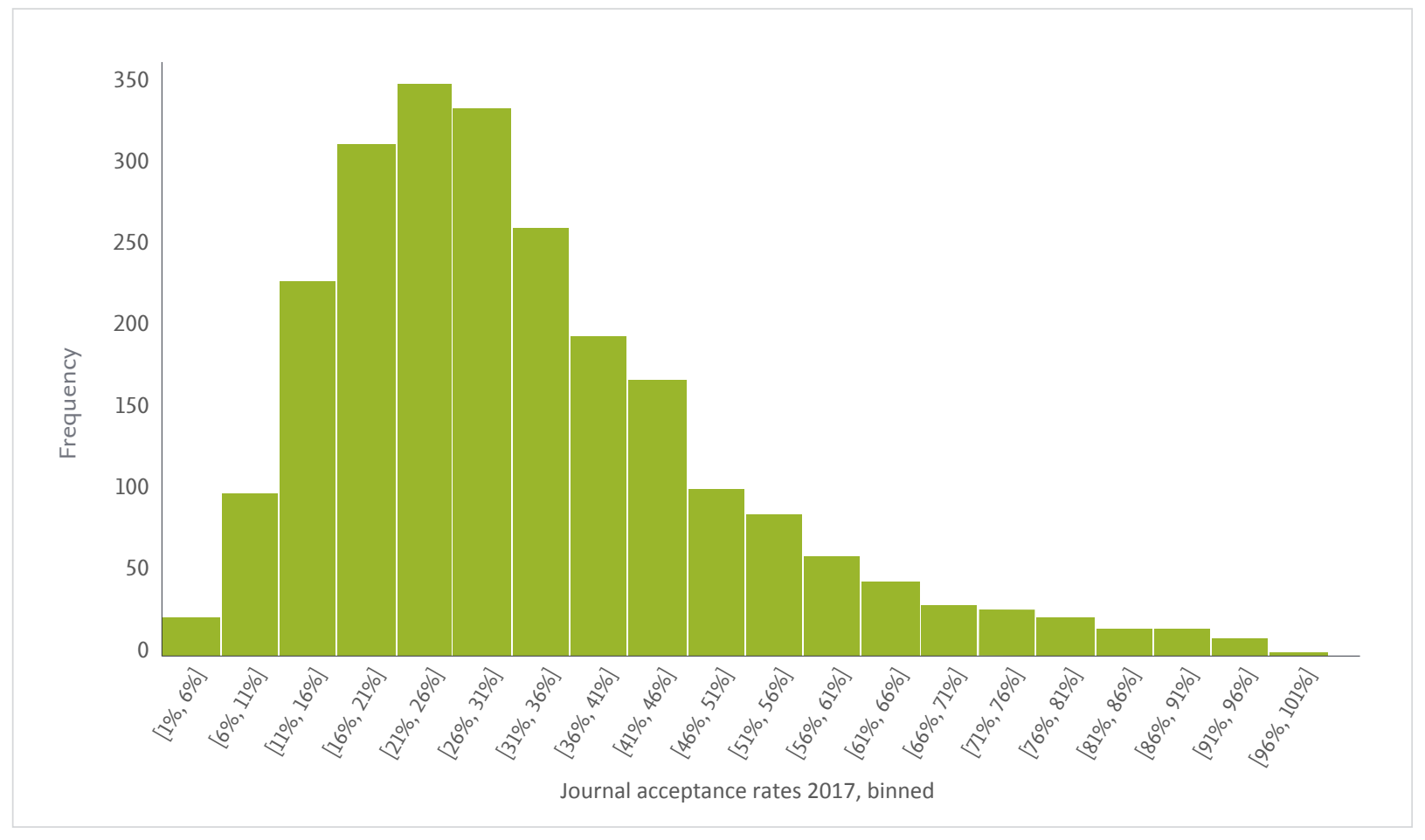

Figure 1: Distribution of 2017 journal acceptance rates for all journals in the dataset $(n=2,371)$. 
Hypothesis: Journals vary in size, yet may be in competition for the same authors and manuscripts. If a small journal feels at risk of being outpaced by a large journal then it may raise its acceptance rates to help make itself appealing to authors.

Overall, there's no strong relationship between journal size and acceptance rate (Figure 2). Sugimoto, Larivière, Ni et al (2013) found that "acceptance increased as the number of articles in a given journal increased." In our data, the relationship between high acceptance rates and small journals is so weak as to be almost non-existent. For small journals (below 100 publications in 2017) in particular, we see that they span the full range of acceptance rates. Larger journals seem to have a slightly smaller, but still wide range of acceptance rates, with most clustered between $10 \%$ and $60 \%$.

\section{Journal age}

Hypothesis: If younger journals are focused on establishing their scope and brand, then their acceptance rates may be higher than older journals.

The journals we studied ranged in age from just-launched to almost 200 years old, and the average age for the journals overall was 26.4 years in 2017. Again, our analysis shows almost no relationship between age and acceptance rates, just a very weak suggestion that older journals tend to have slightly lower acceptance rates (Figure 3).

Sugimoto, Larivière, Ni et al. (2013) previously found that "younger journals tended to have higher acceptance rates." While the very highest acceptance rates do (just) about belong to the younger journals, our data shows journals aged anywhere from 1-60 years with acceptance rates of well over $80 \%$.

\section{Journal citation impact}

Hypothesis: If journal acceptance rates are a signal of journal citation impact or quality, then we might expect a negative correlation between the acceptance rate and a metric such as the field-weighted citation impact (FWCI).

Our data shows no clear relationship between journal citation impact and acceptance rate (Figure 4). There are some high citation impact titles with high acceptance rates and vice versa. The very highest citation impact journals do tend towards lower acceptance rates, but these are journals with FWCI values above 3.0; as the global average for all journals is 1.0, these journals are extremely strong performers. Even then, the acceptance rates tend to be between $\sim 5$ and $40 \%$. So at best, we might say that journals with extremely high impact tend to have acceptance rates of $40 \%$ or below, but that statement hardly seems useful for authors considering where to submit a manuscript. Overall, our data suggest that low acceptance rates aren't a reliable signal of high citation impact; there's just too much variance in the data.

Where we have chosen to compare journal acceptance rates to $\mathrm{FWCl}$, previous research has considered other measures of impact. Frontiers (2015) found "absolutely no correlation between reject rates and impact factor." In contrast to that finding and ours, Sugimoto, Larivière, Ni et al. (2013) found a weak negative correlation between acceptance rate and Journal Impact Factor, and suggested that journals with higher impact might attract more authors, thereby driving up selectivity and acceptance rates.

In the supplementary material available with this report, you'll also find journal acceptance rates plotted against 2018 CiteScore journal metrics, which tells a very similar story to Figure 4. 


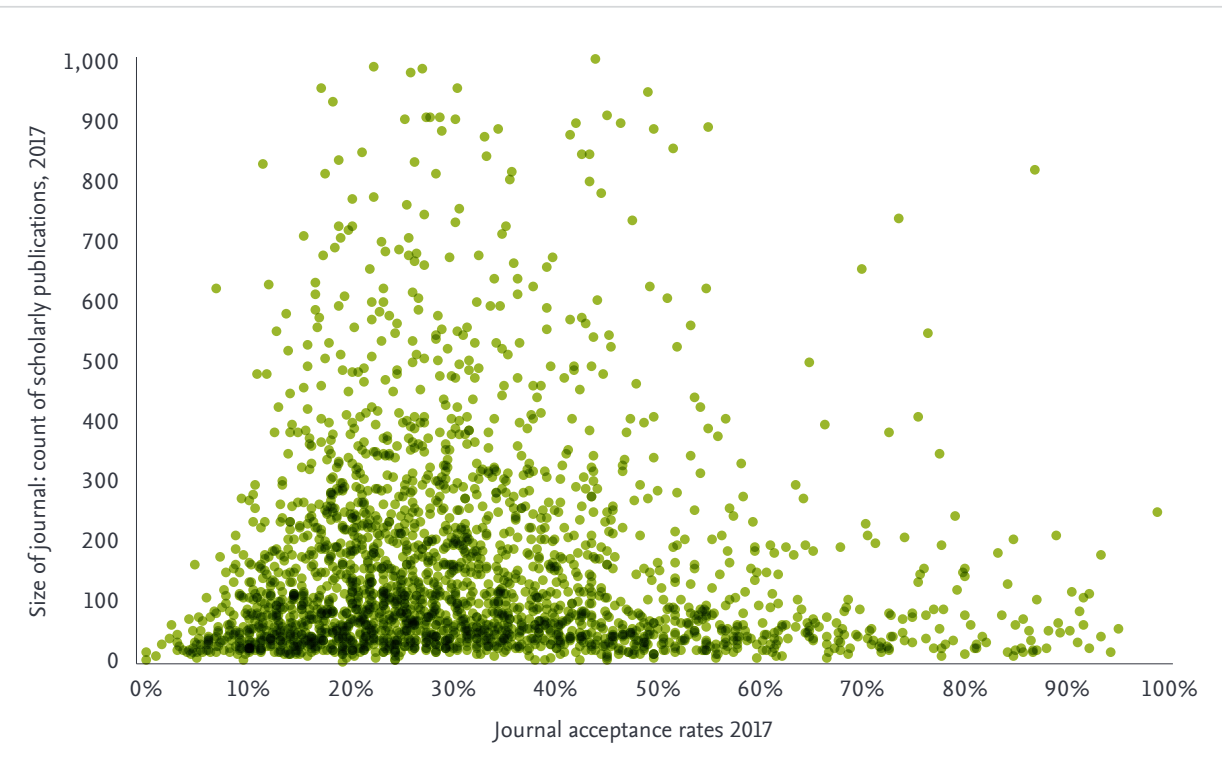

Figure 2: 2017 journal acceptance rates plotted against the size of the journal (count of articles, reviews, conference proceedings papers) in 2017 ( $n=2,371$, but chart limited to show journals with $<1000$ publications, which excludes 50 journals).

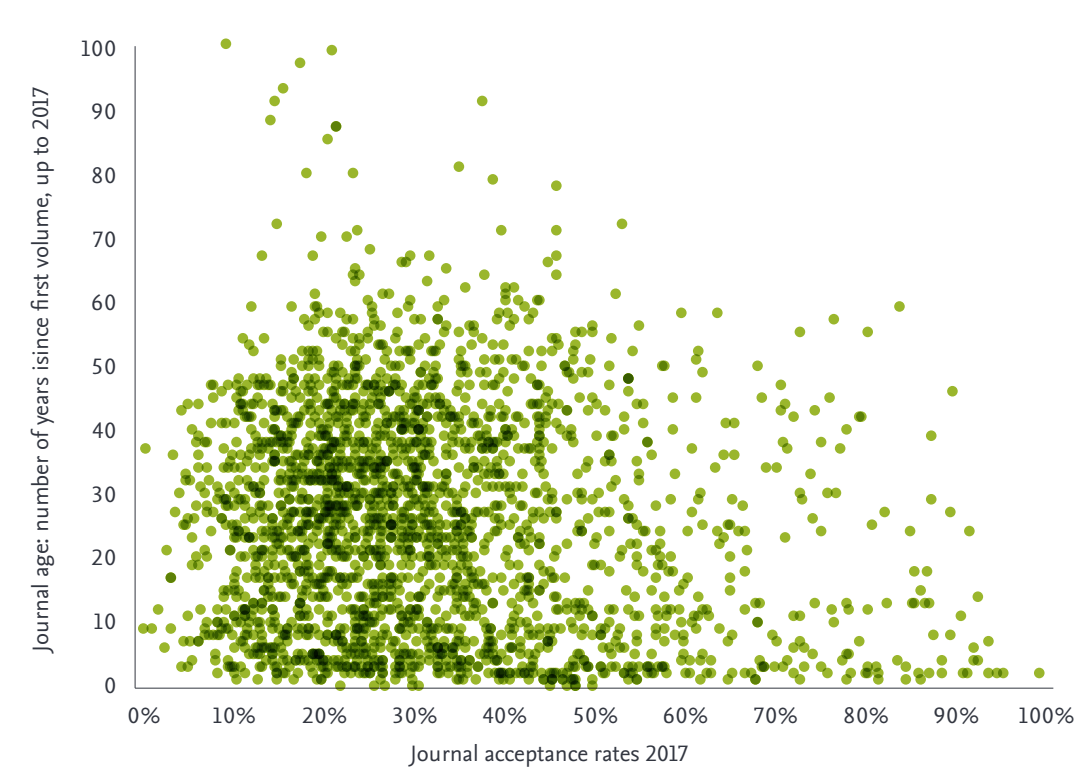

Figure 3: 2017 journal acceptance rates for all journals plotted against the journal age (years since Volume 1 was published) in 2017 ( $n=2,371$, but chart limited to show journals aged 100 years or less, which excludes 11 journals).

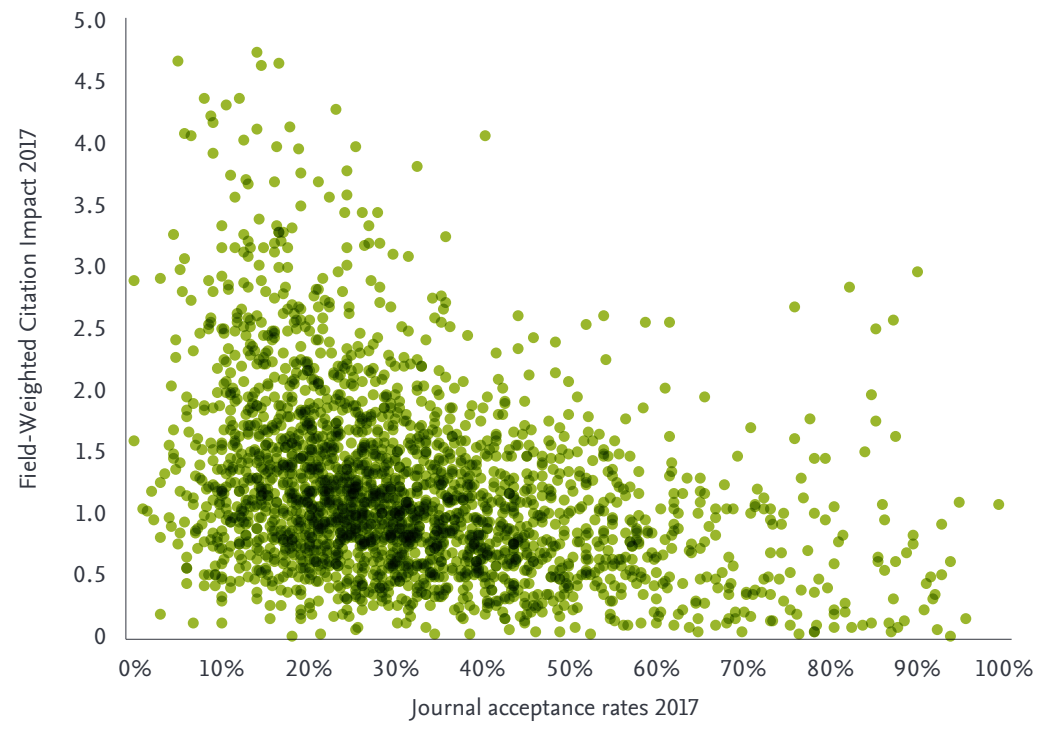

Figure 4: 2017 journal acceptance rates for all journals plotted against the Field-Weighted Citation Impact of the journal in 2017 ( $n=2,371$, but chart limited to show journals with $\mathrm{FWCI}$ of 5 or below, which excludes 21 journals). Field-Weighted Citation Impact measures citation impact across a consistent $0-3$ years after publication and accounts for differences in citation activity by different types of publication, different subject areas, and years of publication; the global average is 1.00 . 
Journal focus: articles

or reviews?

Hypothesis: If a journal publishes a relatively high number of reviews, then on the basis that reviews are typically 'invited' by publications, the journal acceptance rate may be relatively high.

So far, we've grouped all the journals in our dataset together and have considered attributes that an editorial board might not have a lot of control over (age, size of field and/or page budgets), but of course, these journals make choices about what type of research paper they're going to publish. So we compared the review publications as a share of all publications from 2017. This provides a way to identify reviewonly or review-focused journals, which might skew our results (Figure 5) due to the way that some reviews are commissioned and might therefore be less likely to be rejected as being 'out of scope', for example. The chart shows a select few journals (40) with $100 \%$ review articles published in 2017, but even among these journals, the acceptance rates range from $16 \%$ to $89 \%$. Overall, this data shows no correlation between share of review publications and acceptance rate.

\section{Open Access model}

Hypothesis: If open access journals survive financially on article publication charges, then we might expect them to have relatively higher acceptance rates.

The majority of the journals in our dataset offer an open access publication choice to authors ('hybrid open access') with just a few offering no open access ('subscription only') or open access which is not paid by the author ('subsidized open access'). It is those that are 'gold open access' (those that only offer an authorpays immediate open access model) whose results stand out (Figure 6). The other three groups of journals are fairly similar in the distribution of acceptance rates, but the gold open access journals have the highest average acceptance rate (45.9\%). Similarly, Sugimoto, Larivière, Ni et al. (2013) found that "open access journals in all disciplines have significantly higher acceptance rates."

This does suggest that gold open access journals tend to have higher acceptance rates than other journals; something that might follow the logic that these journals are reliant on article publishing charges for their existence. However, it should be noted that these journals are also considerably younger on average than any others ( 7.8 average years' old in 2017, compared to the hybrid open access journals which were 28.6 years' old on average). Only time will tell whether acceptance rates decrease over time as journals age and open access publishing matures, or whether the financial model of the journal will ensure that higher acceptance rates become the norm.

\section{Topical diversity}

Hypothesis: If the scope of a journal is narrow or niche, then its acceptance rate may be relatively low as it needs to be selective in what it publishes.

Some journals are very 'niche' in their topical focus, while others have a broad-ranging scope. Could this have an impact on the acceptance rates? Our research shows that not the case. Most journals have a low-mid range topical diversity of references, but no relationship to acceptance rates is established. See the Methodology section for details on the supplementary data. 


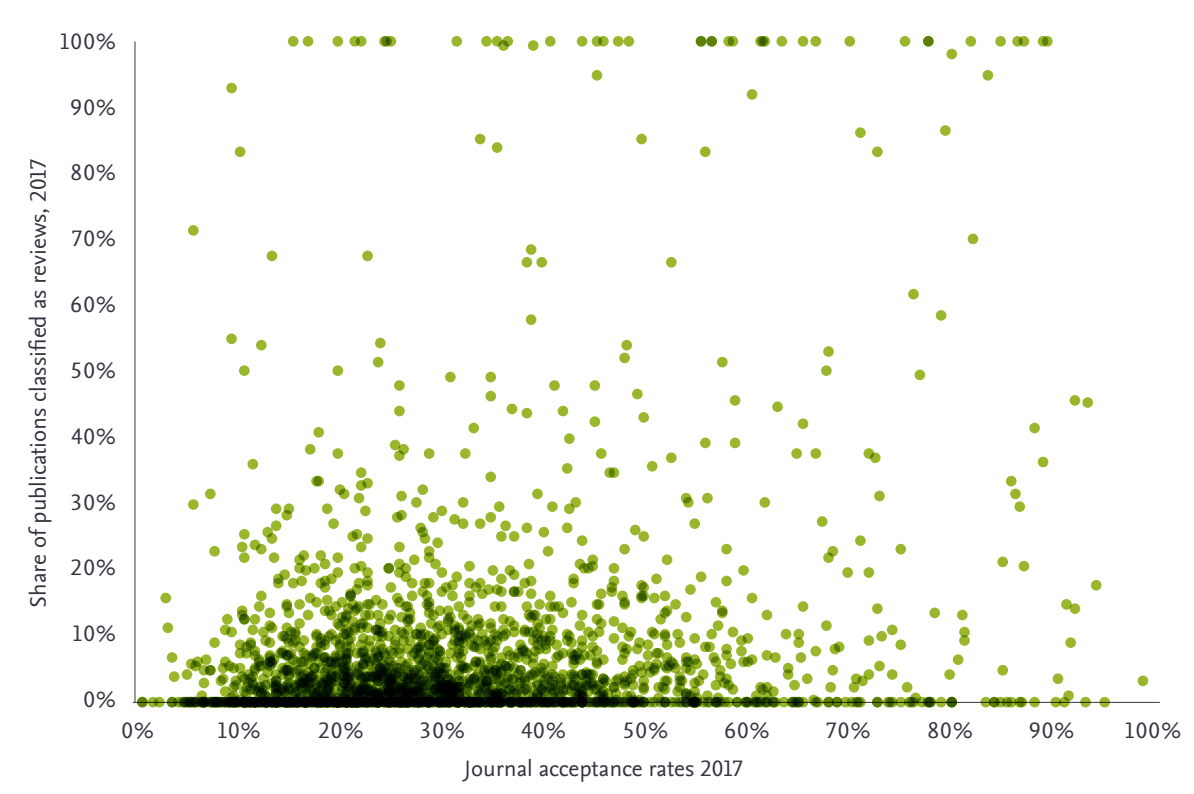

Figure 5: 2017 journal acceptance rates for all journals plotted against the share of Review publications as classified by Scopus among 2017 scholarly publications. The share is of all Articles, Reviews, Conference Proceedings, Short Surveys and Data Papers. $(n=2,371)$

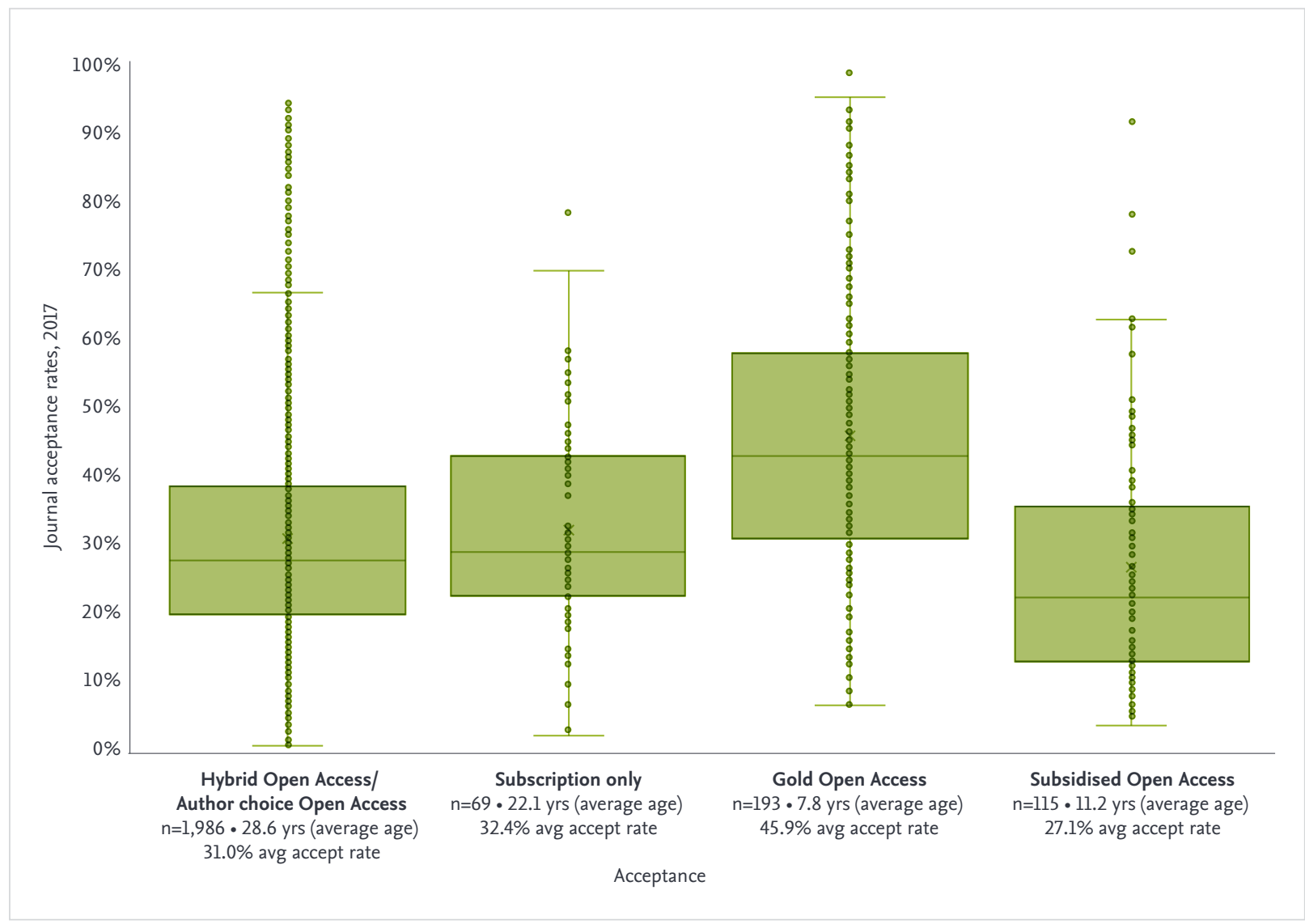

Figure 6: 2017 journal acceptance rates for all journals plotted as box plots, split up by open access model on offer to authors as of 2017. Age is the average for journals per open access model calculated as count of years since Volume 1 in $2017(n=2,371)$ 


\section{Subject area}

Hypothesis: As with many journallevel indicators and metrics, we might expect differences across the various subject areas. Reminder: the journals covered in this report have a good coverage of all fields, with the exception of the social sciences and the arts and humanities.

The last trait we considered was the subject area of the journal. The journal map (Figure 7) shows the coverage of the journals in our dataset and the range of acceptance rates. Certain areas of the map are certainly more inclined towards lower acceptance rates: economics and computer science in particular and, to a slightly lesser extent, mathematics. This might suggest that journals in these formal sciences are tougher to get published in, perhaps driven by a higher degree of 'right' or 'wrong' answers. In contrast, medicine, nursing and life sciences tend to have higher acceptance rates, perhaps reflecting more subjective publishing.

While we have used a different approach, these findings align relatively well with those of Sugimoto, Larivière, Ni et al. (2013), where journals in the health sciences were found to have the highest acceptance rates and those in the business sciences had the lowest. In that paper, competition for publication in business journals was suggested as a reason for this finding. Frontiers (2016) also found that social sciences had relatively high rejection rates.

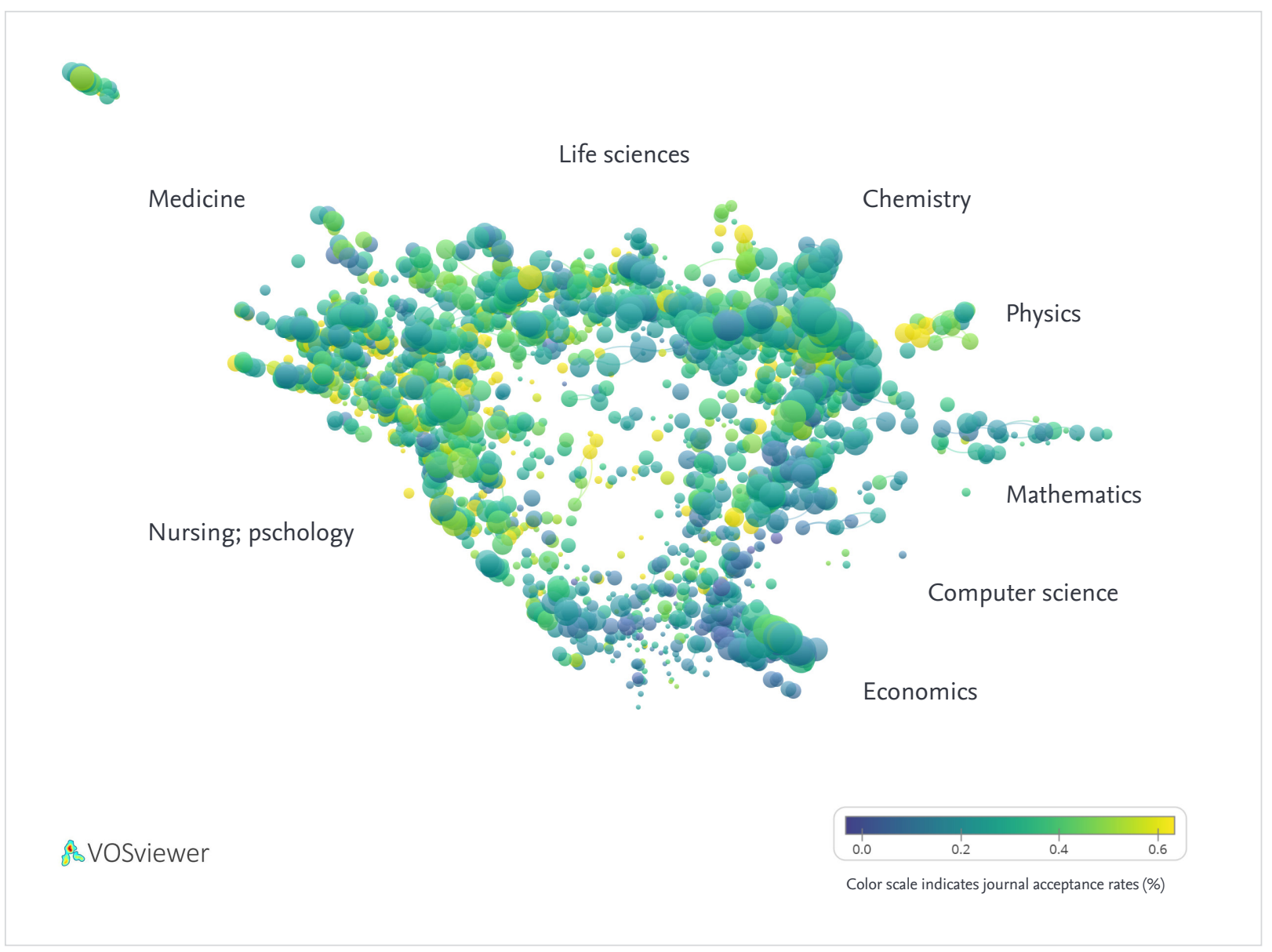

Figure 7: a map of all the journals in the dataset, showing co-citation relationships among publications published by journals between 2012 and 2016, indicating topical similarity. Colors show the acceptance rates: darker blues indicate low acceptance rates, ranging to high acceptance rates in yellow. Node size indicates link strength to other journals. Created using Scopus and VOSviewer. Labels are added manually based on journal names (which have subsequently been removed from each node to maintain anonymity). $(n=2,371)$ 


\title{
Summary
}

\author{
Journal Attribute \\ Relationship to Acceptance Rate \\ Journal size \\ The largest journals tend to have relatively lower acceptance rates compared to smaller \\ journals, but even there the rates range between approx. 10\% and 60\%. \\ Journal age \\ Journal impact \\ Journal publishing \\ Open Access model \\ Topical diversity \\ Journal subject area \\ The oldest journals do tend to have lower acceptance rates than younger journals, but \\ the correlation is weak. \\ Extremely high impact journals tend to show relatively low acceptance rates, but even \\ then there is a lot of variation in the data (acceptance rates still vary between $\sim 5$ and $50 \%$ ). \\ No relationship was identified between the share of review papers published and the \\ acceptance rate. \\ Gold open access journals tend to have higher acceptance rates than other open access \\ models, but these also tend to be the youngest journals. \\ No relationship between the breadth of a journal's scope and the acceptance rate. \\ Journals in the formal sciences (economics, computer science, mathematics) tend to \\ have lower acceptance rates than those in the life sciences and medicine.
}

We identified the fact that low acceptance rates are demonstrated typically by very large, very old and very high impact journals, as well as those that are not 'gold open access.' That's a mixed bag of attributes. The relationship to impact is nuanced and not strong enough for us to state firmly that acceptance rates are a signal of quality or impact. Importantly, we found that even where relationships between journal attributes and acceptance rates could be identified, the variance in the acceptance rate is still so high that the findings are unlikely to be useful in the real world.

So where does that leave authors considering which journals they should submit their manuscript to?
We believe that journal acceptance rates do hold meaning: they indicate to prospective authors the probability of acceptance of their manuscript, based on historical success rates at the same journal. As such, we believe that journal acceptance rates have a place in the array of journal metrics and that they should be made publicly available wherever possible. However, acceptance rate is not a signal of other attributes and so it should be made available alongside other metrics and indicators, but not conflated with them.

Fortunately, that's often already the case. Journal homepages that display acceptance rates often also offer the latest annual journal citation impact metrics, plus information about speed of publication, scope of articles and peer review process. This combination of information is important and useful. The only recommendation we make is for publishers and journals to consider how they present their acceptance rate and to be wary of suggesting this is a signal of impact, quality or stringency. Instead we suggest stating the acceptance rate for what it is: quite simply, the count of manuscripts that were accepted as a share of those submitted in a given period. 



\section{References}

Barber, K. (2019) 2018: Editor's Report-A Year of Engagement. Journal of Cutaneous Medicine and Surgery, 2(2), 145-147. https://doi.org/10.1177/1203475419831304

Brembs, B. (2018) Prestigious Science Journals Struggle to Reach Even Average Reliability. Frontiers of Human Neuroscience, 12(37), https://doi.org/10.3389/ fnhum.2018.00037

Elsevier Journal Finder, accessed 6 November 2019. https://journalfinder.elsevier.com

Elsevier Journal Insights, American Journal of Ophthalmology, accessed 6 November 2019. https:// journalinsights.elsevier.com/journals/o002-9394

Emmer, A. (2019) The careers behind and the impact of solo author articles in Nature and Science. Scientometrics, 120(2)825-840. https://doi.org/10.1007/s11192-019-03145-5

Forero, D.A., Oermann, M.H., Manca, A. et al. (2018) Negative Effects of "Predatory" Journals on Global Health Research. Annals of Global Health, 84(4), 584-589. http:// doi.org/10.29024/aogh.2389

Frontiers (2015) Selecting for impact: new data debunks old beliefs, accessed 6 November 2019 https://blog. frontiersin.org/2015/12/21/4782/
Frontiers (2016) New Data Debunks Old Beliefs: Part 2, accessed 6 November 2019 https://blog.frontiersin. org/2016/03/04/initial-findings-confirmed-no-significantlink-between-rejection-rate-and-journal-impact/

Hall, J.C. (2018) Journal of regional analysis \& policy's 2018 editor's report. Journal of Regional Analysis and Policy, 48(4), 76-77

Manning, W.J. (2017) Review of Journal of Cardiovascular Magnetic Resonance (JCMR) 2015-2016 and transition of the JCMR office to Boston, 19. https://doi.org/10.1186/ s12968-017-0423-x

Metrics Toolkit, accessed 6 November 2019. https://www. metrics-toolkit.org/journal-acceptance-rate

Springer Journal Suggester, accessed 6 November 2019. https://journalsuggester.springer.com/

Sugimoto, C.R., Larivière, V., Ni, C. et al. (2013) Journal acceptance rates: A cross-disciplinary analysis of variability and relationships with journal measures. Journal of Informetrics, 7(4), 897-906. https://doi.org/10.1016/j. joi.2013.08.007 


\section{About the Author}

\section{Rachel Herbert}

Rachel Herbert is a Senior Research Evaluation Manager at Elsevier. She has worked in scholarly publishing for over 10 years and has an active interest in the evaluation of research through the lens of gender. Her most recent major project was Elsevier's Research Futures report, which created scenarios of the future of research and research culture over the coming decade.

https://orcid.org/0000-0002-4088-1223

\section{Acknowledgments}

Thanks go to Alex Ponsford for data curation and Andrew Plume and Kate Gasson for reviewing.

\section{How to cite:}

Herbert, R. Accept me, accept me not: what do journal acceptance rates really mean? ICSR Perspectives (2019)

\section{About the International Center for the Study of Research}

The ICSR's mission is to cultivate the thoughtful use of metrics and indicators in research evaluation and to promote evaluation best practices. The ICSR is tasked with developing, characterizing and validating new and existing research metrics, indicators and research assessment practices; it also supports independent, external studies on topics within this scope.

The ICSR's modus operandi will be to encourage the examination of research using arrays of metrics and qualitative and quantitative methods rather than favoring one-size-fits-all approaches. The proposed arrays will allow researchers and analysts to more effectively evaluate research on dimensions such as productivity, quality, and impact. The Center will draw on the interconnected disciplines of research evaluation, bibliometrics and scientometrics, science of science, science and technology studies, and science of team science.

The ICSR is supported in its work by the ICSR Advisory Board, comprised of experts in research metrics, indicators and research assessment practices drawn from within and beyond Elsevier and selected to reflect a diversity of gender, geography, culture and research expertise.
Learn more and sign up for email alerts: www.elsevier.com/icsr

@IntCtrStudyRes 